\title{
Acknowledgement to reviewers in 2015
}

Yasuo Ogawa

\section{Abstract}

Contributing reviewers: The Editorial Board of Earth, Planets and Space wishes to thank the following reviewers for their help in evaluating papers for the year 2015.

\begin{tabular}{l} 
Mangalathayil Abdu \\
Brazil \\
Lucilla Alfonsi \\
Italy \\
Jason Ali \\
Hong Kong \\
Patrick Alken \\
United States of America \\
Patrick Alken \\
United States of America \\
Takanobu Amano \\
Japan \\
Hiroki Ando \\
Japan \\
Ryosuke Ando \\
Japan \\
SeyedMasoud Ansari \\
Canada \\
Victoria Antoci \\
Denmark \\
Graham Appleby \\
United Kingdom \\
Tohru Araki \\
Japan \\
Claudia Arango-Galván \\
Mexico \\
Christina Arras \\
Germany \\
Anasuya Aruliah \\
United Kingdom \\
Ayumi Asai \\
Japan \\
Kazushi Asamura \\
Japan \\
\hline
\end{tabular}

Betul Aytorre

Turkey

Toshitaka Baba

Japan

Kiyoshi Baba

Japan

Nikolai Bagdassarov

Germany

Karsten Bahr

Germany

Nanan Balan

United Kingdom

Georgios Balasis

Greece

Maria Ana Baptista

Portugal

Sylvain Barbot

Singapore

Serif Baris

Turkey

Yuri Barkin

Russian Federation

William Barnhart

United States of America

Yusuf Bayrak

Turkey

Chris Bean

Ireland

Pierre Beck

France

Luther Beegle

United States of America

Ciaran Beggan

United Kingdom
Alec James Bennett

United Kingdom

Paolo Bergamo

United Kingdom

David Berube

United States of America

Naoki Bessho

United States of America

Mukunda Bhattarai

Nepal

Johannes Böhm

Austria

Olawale Bolajji

Nigeria

Alessandro Bonaccorso

Italy

Luis-Fabian Bonilla

France

John Booker

United States of America

Joe Borovsky

United States of America

David Boteler

Canada

Eran Bouchbinder

Israel

Urban Brändström

Sweden

Gianni Bressan

Italy

Maxwell Brown

Germany

Patrick Brown

United Kingdom

Correspondence: editor-in-chief@earth-planets-space.org

Earth Planets and Space, Volcanic Fluid Research Center, Tokyo Institute of

Technology, Tokyo, Japan

Springer

(C) 2016 Ogawa. Open Access This article is distributed under the terms of the Creative Commons Attribution 4.0 International License (http://creativecommons.org/licenses/by/4.0/), which permits unrestricted use, distribution, and reproduction in any medium, provided you give appropriate credit to the original author(s) and the source, provide a link to the Creative Commons license, and indicate if changes were made. 
Colin Brown

Ireland

Maria Teresa Brunetti

Italy

Stephan Buchert

Sweden

Aaron Burton

United States of America

Andrew Calvert

Canada

Antonio Camacho

Spain

Juan Pablo Canales

United States of America

Claudia Candido

Brazil

Elisabeth Canet

Switzerland

Carlo Cauzzi

Switzerland

C. David Chadwell

United States of America

Chung-Han Chan

Singapore

Kevin Chao

United States of America

Alan Chave

United States of America

Chia-Hung Chen

Taiwan

Kate Huihsuan Chen

Taiwan

Peter Chi

United States of America

Taejin Choi

Republic of Korea

Arnaud Chulliat

United States of America

Pierre Cilliers

South Africa

Edward Cliver

United States of America

Martin Connors

Canada

Catherine Constable

United States of America

Dragos Constantinescu

Romania

Luca Crescentini

Italy

Phil Cummins

Australia

Louis De Barros

France
Angelo De Santis

Italy

Mark Dekkers

Netherlands

Mitsue Den

Japan

Raffaele Di Stefano

Italy

Catherine Dieval

United Kingdom

Sait Dikmen

Turkey

Henryk Dobslaw

Germany

Danan Dong

China

Douglas Dreger

United States of America

Thomas Driesner

Switzerland

Nelia Dunbar

United States of America

Eric Dunham

United States of America

Firdevs Duru

United States of America

Donna Eberhart-Phillips

United States of America

Yusuke Ebihara

Japan

Itai Einav

Australia

Ana Elias

Argentina

Oliver Ernst

Germany

Eileen Evans

United States of America

Yuichiro Ezoe

Japan

Thomas Farges

France

Bulut Fatih

Turkey

Lujia Feng

Singapore

Graziano Ferrari

Italy

Mei-Ching Fok

United States of America

Colin Forsyth

United Kingdom

Nico Fournier

New Zealand
William Frank

United States of America

Marc Fries

United States of America

Mathias Fritsche

Germany

Yuning Fu

United States of America

Ikuko Fujii

Japan

Shigeru Fujita

Japan

Yukitoshi Fukahata

Japan

Yoichi Fukuda

Japan

Koji Fukuma

Japan

Stephen Fuselier

United States of America

Christine Gabrielse

United States of America

Damien Gaudin

Italy

Essam Ghamry

Egypt

Nicolas Gillet

France

Ronald Gilliland

United States of America

Jeffrey Gillis-Davis

United States of America

Alex Glocer

United States of America

Mareike Godolt

Germany

Ethem Gorgun

Turkey

Tada-nori Goto

Japan

Raphael Grandin

France

Ronni Grapenthin

United States of America

Robert Graves

United States of America

Richard Gross

United States of America

Philippe Gueguen

France

Amitava Guharay

Brazil

Levent Gülen

Turkey 


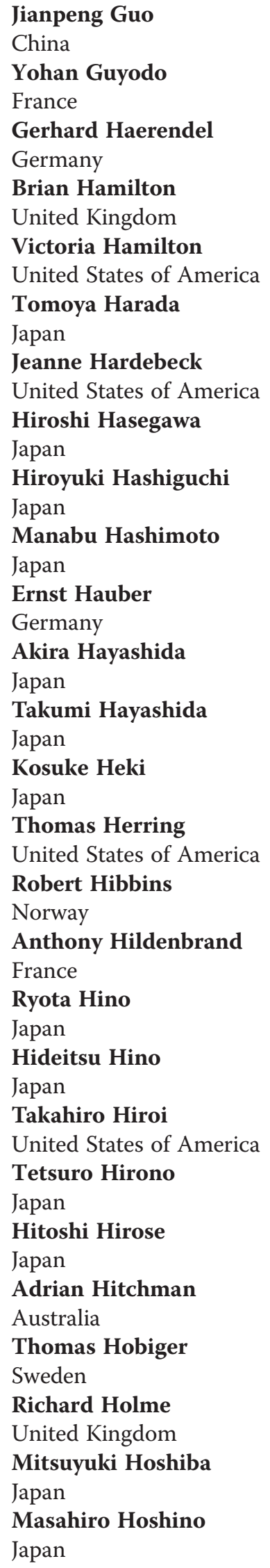

\begin{tabular}{|c|c|}
\hline Keisuke Hosokawa & Mike Jackson \\
\hline Japan & United States of America \\
\hline Susan Hough & Kishor Jaiswal \\
\hline United States of America & United States of America \\
\hline Heidi Houston & Pekka Janhunen \\
\hline United States of America & Finland \\
\hline Yi-Ling Huang & Chun-Sung Jao \\
\hline Taiwan & Taiwan \\
\hline Qinghua Huang & Dominique Jault \\
\hline China & France \\
\hline Chaosong Huang & Shuanggen Jin \\
\hline United States of America & China \\
\hline Hsin-Hua Huang & Hidekatsu Jin \\
\hline United States of America & Japan \\
\hline Benoit Hubert & Romain Jolivet \\
\hline Belgium & United Kingdom \\
\hline Guang-Pu Huo & Arthur Jolly \\
\hline China & New Zealand \\
\hline Giampiero Iaffaldano & Neli Jordanova \\
\hline Australia & Bulgaria \\
\hline Hiroshi Ichihara & Yoshihiro Kakinami \\
\hline Japan & Japan \\
\hline Akimasa Ieda & Yavor Kamer \\
\hline Japan & Switzerland \\
\hline Takeshi Iinuma & Junzo Kasahara \\
\hline Japan & Japan \\
\hline Yoshihisa Iio & Aitaro Kato \\
\hline Japan & Japan \\
\hline Steve Ikubanni & Teruyuki Kato \\
\hline Nigeria & Japan \\
\hline Shinsuke Imada & Kei Katsumata \\
\hline Japan & Japan \\
\hline Makoto Inoue & Bülent Kaypak \\
\hline Japan & Turkey \\
\hline T. Serkan Irmak & Takahito Kazama \\
\hline Turkey & Japan \\
\hline Takeo Ishibe & Kunihiro Keika \\
\hline Japan & Japan \\
\hline Tetsuro Ishida & Lindsay Keller \\
\hline Japan & United States of America \\
\hline Takemi Ishihara & Ben Kennedy \\
\hline Japan & New Zealand \\
\hline Mamoru Ishii & Brian Kennett \\
\hline Japan & Australia \\
\hline Naoto Ishikawa & Alam Kherani \\
\hline Japan & Brazil \\
\hline Yuzo Ishikawa & Takashi Kikuchi \\
\hline Japan & Japan \\
\hline Osamu Ishizuka & Hyosub Kil \\
\hline Japan & United States of America \\
\hline Bambang Istadi & Kwang-Hee Kim \\
\hline Indonesia & Republic of Korea \\
\hline Toshihiko Iyemori & Takenari Kinoshita \\
\hline Japan & Japan \\
\hline
\end{tabular}


Stephen Kirby

United States of America

Kentarou Kitamura

Japan

Tomokazu Kobayashi

Japan

Argun Kocaoglu

Turkey

Shuichi Kodaira

Japan

Kazuki Koketsu

Japan

Masao Komazawa

Japan

Hisao Kondo

Japan

Michael Korn

Germany

Michael Kosch

South Africa

Stavros Kotsiaros

Denmark

Sasha Koustov

Canada

Svetlana Kovacikova

Czech Republic

Jan Krynski

Poland

Karel Kudela

Slovakia

Keiko Kuge

Japan

Sanjay Kumar

India

Sushil Kumar

Fiji

Li-Wei Kuo

Taiwan

Kiyoshi Kuramoto

Japan

Shigekazu Kusumoto

Japan

Takao Kuwabara,

Japan

Simon Lamb

New Zealand

Luca Lanci

Italy

Falko Langenhorst

Germany

Benoit Langlais

France

Juan Larrasoaña

Spain
Ya-ting Lee

Taiwan

Vincent Lesur

Germany

Yongxiang Li

China

Zhao Li

China

Yongxiang Li

China

Guozhu Li

China

Sanjay Limaye

United States of America

Ting-Li Lin

Taiwan

Chen-Hong Lin

Taiwan

Eric Lindsey

United States of America

Eric Lindsey

Singapore

Libo Liu

China

Yajing Liu

Canada

Huixin Liu

Japan

Qingsong Liu

China

Jianjun Liu

China

Yonghua Liu

China

Jing Liu

China

Libo Liu

China

Ruifeng Liu

China

Maureen Long

United States of America

Jeffrey Love

United States of America

Jack Loveless

United States of America

Frank Lowes

United Kingdom

Hermann Lühr

Germany

Sonya Lyatskaya

United States of America

Susan Macmillan

United Kingdom
Yuta Maeda

Japan

Fukashi Maeno

Japan

Alessia Maggi

France

Martin Mai

Saudi Arabia

Zinovy Malkin

Russian Federation

Ian Mann

Canada

Carlos Martinis

United States of America

Takashi Maruyama

Japan

Larry Mastin

United States of America

Robin Matoza

United States of America

Makoto Matsubara

Japan

Mitsuru Matsumura

Japan

Ayako Matsuoka

Japan

Toru Matsuzawa

Japan

Andrew McDonald

Australia

Andrew McGonigle

United Kingdom

Steven McNutt

United States of America

Mustapha Meghraoui

France

Thomas Meier

Germany

Irwan Meilano

Thailand

Michael Meindl

Switzerland

Jose Merayo

Denmark

Amalia Meza

Argentina

Ingo Michaelis

Germany

Steve Milan

United Kingdom

Ralph Milliken

United States of America

Toshiaki Mishima

Japan 
Yuji Mitsuhata

Japan

Yayoi Miura

Japan

Masaaki Miyahara

Japan

Yukinaga Miyashita

Japan

Takahiro Miyauchi

Japan

Gregory Moore

United States of America

James Mori

Japan

Tetsuo Motoba

Japan

Jon Mound

United Kingdom

Dietmar Muller

Australia

Kazuoki Munakata

Japan

Hiroshi Munekane

Japan

Victor Munoz

Chile

Hideki Murakami

Japan

Masayuki Murase

Japan

Isao Murata

Japan

Yasuhiro Murayama

Japan

Kyle Murphy

United States of America

Giovanni Muscari

Italy

Tomoo Nagahama

Japan

Nandini Nagarajan

India

Tsutomu Nagatsuma

Japan

Manoj Nair

United States of America

Tomoko Nakagawa

Japan

Haruhisa Nakamichi

Japan

Mamoru Nakamura

Japan

Takeshi Nakamura

Japan
Tadas Nakamura

Japan

Tomoki Nakamura

Japan

Masaru Nakano

Japan

Shinya Nakano

Japan

Shun Nakano

Japan

Yasuhito Narita

Austria

Keri Nicoll

United Kingdom

Junsheng Nie

China

Takafumi Niihara

Japan

Hiroshi Nishi

Japan

Kiwamu Nishida

Japan

Yukitoshi Nishimura

United States of America

Takuya Nishimura

Japan

Michi Nishioka

Japan

Fenglin Niu

United States of America

Ran Nof

Israel

Masahito Nose

Japan

Kenji Notsu

Japan

Motoharu Nowada

Japan

Koichiro Obana

Japan

Paul O'Brien

United States of America

Oliver Obrou

Côte D'Ivoire

Giovanni Occhipinti

France

Hitoshi Oda

Japan

Tsutomu Ogawa

Japan

Masaki Ogawa

Japan

Takeshi Ohba

Japan
Takahiro Ohkura

Japan

Takao Ohminato

Japan

Masao Ohno

Japan

Michihiro Ohori

Japan

Tsuneo Ohsumi

Japan

Yusaku Ohta

Japan

Shinichi Ohtani

United States of America

Hiroyo Ohya

Japan

Kyoko Okino

Japan

Nils Olsen

Denmark

Yoshiharu Omura

Japan

Kyosuke Onishi

Japan

Gonca Orgulu

Turkey

Naoto Oshiman

Japan

Yuichi Otsuka

Japan

Michio Otsuki

Japan

Laurianne Palin

Sweden

Evgeny Panov

Austria

Vladimir Papitashvili

United States of America

Jisun Park

United States of America

Jin-Oh Park

Japan

Greig Paterson

China

Erricos Pavlis

United States of America

Josef Pek

Czech Republic

Efim Pelinovsky

Russian Federation

Tom Pering

United Kingdom

Vera Pessina

Italy 


\author{
Anatoli Petrukovich \\ Russian Federation \\ Anatoly Petukhin \\ Japan \\ Slava Pilipenko \\ Russian Federation \\ Beatrice Pinel-Puyssegur \\ France \\ Risto Pirjola \\ Canada \\ Pavlo Ponomarenko \\ Canada \\ William Power \\ New Zealand \\ Paul Prikryl \\ Canada \\ Philip Pritchett \\ United States of America \\ Shishir Priyadarshi \\ Poland \\ Christoph Puethe \\ Switzerland \\ Michael Purucker \\ United States of America \\ Huafeng Qin \\ China \\ Babatunde Rabiu \\ Nigeria \\ Marcin Rajner \\ Poland \\ Penki Ramesh Kumar \\ India \\ Jeanne Rasson \\ Belgium \\ Nick Rawlinson \\ United Kingdom \\ Zhengyong Ren \\ China \\ Paul Richards \\ Colombia \\ Minako Righter \\ United States of America \\ Adam Ringler \\ United States of America \\ Maurizio Ripepe \\ Italy \\ Julie Roberge \\ Mexico \\ Andrew Roberts \\ Australia \\ Thomas Rockwell \\ United States of America \\ Alan Rodger \\ United Kingdom
}

Arthur Rodgers

United States of America

Evelyn Roeloffs

United States of America

Diana Roman

United States of America

Martin Rother

Germany

Zafeiria Roumelioti

Greece

Eugene Rozanov

Switzerland

Andrei Runov

United States of America

Timo Saarinen

Finland

Terence Sabaka

United States of America

Erdal Safak

Turkey

Susumu Saito

Japan

Takeshi Saito

Japan

Tatsuhiko Saito

Japan

Shin'Ichi Sakai

Japan

Tetsuya Sakai

Japan

Kazuyo Sakanoi

Japan

Tetsuya Sakuyama

Japan

Vadim Saltykov

Russian Federation

Marilia Samara

United States of America

Kenji Satake

Japan

Natsuo Sato

Japan

Toshimi Satoh

Japan

Heather Savage

United States of America

Romy Schlögel

Italy

David Schriver

United States of America

Gopi Seemala

India

Mamoru Sekido

Japan
Hiromu Seko

Japan

Victor Sergeev

Russian Federation

Robert Shcherbakov

Canada

Chiao-Yao She

United States of America

David Shelly

United States of America

Takuo Shibutani

Japan

Hidetoshi Shibuya

Japan

Tian-Yuan Shih

Taiwan

Hisayoshi Shimizu

Japan

Hiroyuki Shinagawa

Japan

Atsuki Shinbori

Japan

Hiroshi Shinohara

Japan

Kazuo Shiokawa

Japan

Katsuhiko Shiomi

Japan

Masanobu Shishikura

Japan

Masafumi Shoji

Japan

Hugo Gonçalves Silva

Portugal

Weerachai Siripunvaraporn

Thailand

Mikhail Sitnov

United States of America

Maxim Smirnov

Finland

Deborah Smith

United States of America

Stanley Solomon

United States of America

Svenja Sommer

Germany

Sergey Stanchits

United States of America

Robert Stern

United States of America

Joseph Stoner

United States of America

André Stumpf

France 


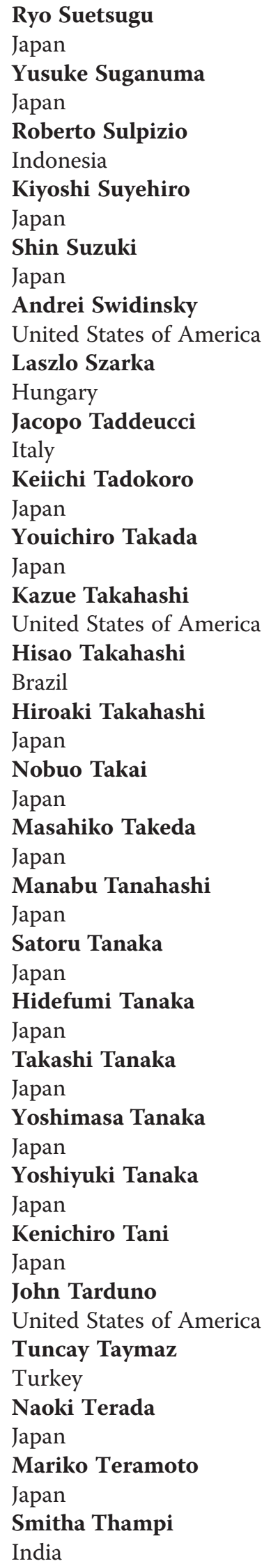

Cristina Thomas

United States of America

Alan Thomson

United Kingdom

Dmitri Titov

Germany

Shinji Toda

Japan

Lars Tøffner-clausen

Denmark

Frank Toffoletto

United States of America

Hiroaki Toh

Japan

Kazushige Tomeoka

Japan

Akihiko Tomiya

Japan

Xiaopeng Tong

United States of America

J. Miquel Torta

Spain

John Townend

New Zealand

Genti Toyokuni

Japan

Oleg Troshichev

Russian Federation

Takuo Tsuda

Japan

Kae Tsunematsu

Japan

Fumiaki Tsunomori

Japan

Bruce Tsurutani

United States of America

Hiroaki Tsushima

Japan

Robert Tyler

United States of America

Naoki Uchida

Japan

Takahiko Uchide

Japan

K. Unnikrishnan

India

Koji Uno

Japan

Teiji Uozumi

Japan

Yoichi Usui

Japan

Hideyuki Usui

Japan
Murat Utkucu

Turkey

Rami Vainio

Finland

Greg Valentine

United States of America

Heikki Vanhamaki

Finland

José Vaquero

Spain

Peter Varga

Hungary

Fabio Vargas

United States of America

Vaclav Vavrycuk

Czech Republic

Ari Viljanen

Finland

Fátima Viveiros

Portugal

Ioannis Vogiatzis

Greece

Greg Waite

United States of America

Yanbin Wang

China

William Ward

Canada

Ingo Wardinski

Germany

Shingo Watada

Japan

Shigeto Watanabe

Japan

Tomo-Hiko Watanabe

Japan

Brenton Watkins

United States of America

Clare E. J. Watt

United Kingdom

Kornyanat Watthanasangmechai Japan

Matt Wei

United States of America

Cynthia Werner

United States of America

Viktor Wesztergom

Hungary

Richard Willingale

United Kingdom

Othon Winter

Brazil

Qian Wu

United States of America 
Zhonghua Xu

United States of America

Peiliang Xu

Japan

Yasuo Yabe

Japan

Hikaru Yabuta

Japan

Yuji Yagi

Japan

Masumi Yamada

Japan

Hidekazu Yamamoto

Japan

Yuhji Yamamoto

Japan

Masa-yuki Yamamoto

Japan

Keiko Yamamoto

Japan

Hiroaki Yamanaka

Japan

Ken'Ichi Yamazaki

Japan
Tao Yang

China

Nobumitsu Yokoi

Japan

Tatsuhiro Yokoyama

Japan

Kiyoshi Yomogida

Japan

Satoru Yoshida

Japan

Mitsuhiro Yoshimoto

Japan

Kazunori Yoshizawa

Japan

Shui-Beih Yu

Taiwan

Yongjae Yu

Republic of Korea

Tao Yuan

United States of America

Yunbin Yuan

China

Han Yue

United States of America
Xinan Yue

China

Akira Sessai Yukimatu

Japan

Yohei Yukutake

Japan

Elena Zanella

Italy

Seiji Zenitani

Japan

Donghe Zhang

China

Yongxian Zhang

China

Xixi Zhao

China

Li Zhao

Taiwan

Shasha Zou

United States of America 